\author{
Military Technical College \\ Kobry El-kobbah, \\ Cairo, Egypt
}

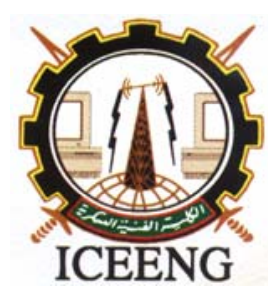

\title{
A SOFTWARE PROGRAM FOR HARMONIC IDENTIFICATION USING GENETIC ALGORITHM
}

\author{
Sherif B. Amer* \\ Hosam K.M. Youssef**, Member, IEEE \\ M.M. Abdel Aziz**, Senior \\ Member, IEEE
}

\begin{abstract}
This paper introduces a software program written in Matlab programming language for the identification and analysis of harmonics in power systems using the Genetic Algorithm (GA) as an optimization technique. The program overcomes a major pitfall of the Fast Fourier Transform (FFT); the spectral leakage. Simulated waveforms were used to test the program. The results obtained were in the form of the Fourier series (magnitudes and phases) with high accuracy.
\end{abstract}

KEYWORDS: Harmonics, Genetic Algorithm, FFT.

\section{INTRODUCTION}

The spread use of non-linear loads such as AC/DC Converters, Static Var compensators and Arc furnaces which draw a non-sinusoidal current resulted in the distortion of the voltage waveforms of power network buses. The harmful effect of harmonics includes overheating in the transformers, cables and rotating machines that reduces the life time of such equipment; telephone interference; improper operation of protective relays; and neutral line overloading due to the zero-sequence nature of the triplen harmonics. So the need of harmonic elimination arises, but first the harmonics spreading in the power network have to be determined and analyzed. There is more than one method for this purpose. The use of harmonic analyzers or spectrum analyzers devices which display the harmonic content (magnitudes and phases) is one of the methods. Although this method is highly reliable it requires on-line continuous measurements and large number of analyzers to be installed to the network. Performing harmonic load flow to identify the steady state voltages at the network buses is among the methods used for harmonic analysis in power systems.

* National Energy Control Center (NECC).

** Electric power and machine Dept., Faculty of Engineering, Cairo University, Egypt 
But still the numerical convergence, long execution time and the need for accurate modeling of harmonic sources and system components among the difficulties facing this method.

Refs [1-4] are examples of using harmonic load flow in harmonic identification. In recent years, Artificial Intelligence (AI) Techniques have gained wide spread popularity in different fields due its simplicity, accuracy and applicability. Naturally, several AI techniques have been used in the field of harmonic identification. Ref [6] uses a combination of least square errors for amplitude estimation and GA for phase estimation. Ref [7] uses GA to obtain the magnitudes of different waveforms. Ref [8] applies the neural network for real time harmonic evaluation.

GAs have recently received much attention as robust stochastic search algorithms for optimization problems [7]. GAs have been applied to various power system problems with promising results $[9,10]$.

This paper introduces a new method based on GA for harmonic identification.

\section{EXPLAINING THE HARMONIC IDENTIFICATION PROBLEM}

The Fourier series for a voltage waveform is in the form:

$$
V(t)=V_{\mathrm{o}}+\sum_{i=1}^{N} V_{s i}(t) \operatorname{Sin}\left(i \omega t+\varphi_{s i}\right)
$$

where $i$ is the harmonic order, $V_{o}$ is the dc component, $V_{s i}$ is the voltage magnitude of the $i^{\text {th }}$ harmonic component, $\varphi_{s i}$ is the phase of the corresponding $i^{\text {th }}$ voltage component and $N$ is the highest harmonic order in the considered study.

From Eq.1 the no. of voltage magnitude unknowns is $(N+1)$, while the no. of phase unknowns is $N$, the total no. of unknowns is then $(2 N+1)$. The proposed program introduces a method for identifying these unknowns using Genetic Algorithm in order to identify the harmonic contents of the voltage waveform.

\section{INTRODUCTION TO GENETIC ALGORITHM}

Genetic Algorithms are a search methods based on the principles of natural selection. The GAs have the ability to solve non-polynomial problems. The GAs begin by generating random solutions known as chromosomes each of them have an evaluation value or fitness. According to Darwin's theory: The solution of better fitness has a higher chance to survive. The basic GAs operators, crossover and mutation, are used to produce more fit chromosomes. The Elitism can be used for automatic copying of the best chromosomes for the next iteration or generation. The same operators are applied to the next generation. The Algorithm stops either at a predefined maximum no. of generations or when the accuracy requirement is achieved. Ref [5] gives a basic introduction to Genetic Algorithms.

\section{GA OPERATORS}


The GAs apply different operators during the search for the optimum solution. These operators are discussed briefly in this section.

Encoding: Encoding of a chromosome is the first thing made when solving a problem with GA. The chromosome is a string containing all of the unknowns in a problem. Fig. 1 shows the construction of the chromosome used in the program. The chromosome consists of two strings: the voltage magnitude and the phase.

\begin{tabular}{ll|l|l|l|l|}
\hline $\mathrm{Vo}_{0}\left|\mathrm{~V}_{1}\right|$ & $\mathrm{V}_{\mathrm{H}}$ & $\Phi_{1} \mid \Phi_{2}$ & $\mid \Phi_{\mathrm{H}}$ \\
\hline $\mathrm{N}+1$ & $\rightarrow$ & $-\mathrm{N} \rightarrow$
\end{tabular}

Fig.1. The construction of a chromosome used in the program

In the developed program real valves encoding is used. The length of the chromosome equals $(2 N+1)$ bits.

Selection: Is to select 2 parents (chromosomes) from the mating pool to produce new offspring (new chromosomes). The mating pool has to be formed to give the best chromosomes a high chance to be selected. The rank selection method is applied in the program. The mating pool is formed for a hundred chromosomes as follows, the best one will have 100 positions in the mating pool, and the worst chromosome will have only one position in the pool.

Crossover: After the selection is done, single-point crossover is made by choosing random position in each chromosome and then swapping the bits that exist after this position. Crossover is applied for the voltage magnitudes and the phases individually.

Mutation: Mutation is performed to prevent falling into a local optimum of the solved problem. Mutation depends on the encoding of chromosomes. Since the developed program uses real values, so mutation is done by adding a small random value to the chromosome randomly chosen bit. Since the chromosome consists of two types of values (voltage magnitude $\&$ phases), so two different values were chosen and added, one for the voltage magnitude string and the other for the phase string.

A modified mutation was applied in the developed program. Suppose a hundred iterations or generations are performed in the program. These generations are then divided into stages (for example, from 1 to 20 then from 21 to 40 and so on). At the first set of generations the error is usually big so a relatively big number is to be added during the mutation process, and then this number is decreased at the followed stages while the error is decreasing.

Elitism: Is made by copying the best chromosomes to the new generation to prevent losing the best one.

\section{GA APPLICATION TO HARMONIC IDENTIFICATION}

A computer program was developed to work on discrete samples of the waveform taken by Analog to Digital converter (A/D) with a sampling frequency $(F s)$. These samples are then supplied to the computer as an array with dimension $N s$, where $N s$ is the no. of samples. The 
program then generates different sets of random variables. Each set is called a chromosome which contains all the unknown variables required in Eq.1.

The randomly generated values of the variables in each chromosome are then substituted in Eq.1 at each of the sampled instances ( $N s$ instances) and the obtained voltage value is subtracted from the measured value of the voltage at the corresponding time instances.

These results are in an error array (Es) of dimension $N s$. To evaluate the fitness for each chromosome, the error array $(E s)$ has to be converted to a single error value. The developed program gives the user the choice between 2 options [7]: the first is to take the absolute maximum error (Emax) in the array where the fitness function $(F)$ will be:

$$
F=1 /(|\operatorname{Emax}|+\alpha)
$$

where $\alpha$ is a very small positive number preventing the fitness for being infinity. The second option is presenting the fitness function $(F)$ in terms of (Esum):

$$
\text { Esum }=\sum_{i=1}^{N s}|E i| / N s
$$

where $E i$ is the $i^{\text {th }}$ element in $(E s)$. Then the fitness $F$ equals:

$$
F=1 /(\mid \text { Esum } \mid+\alpha)
$$

Fig. 2 Represents a flowchart for the general steps used in the formulation of GA.

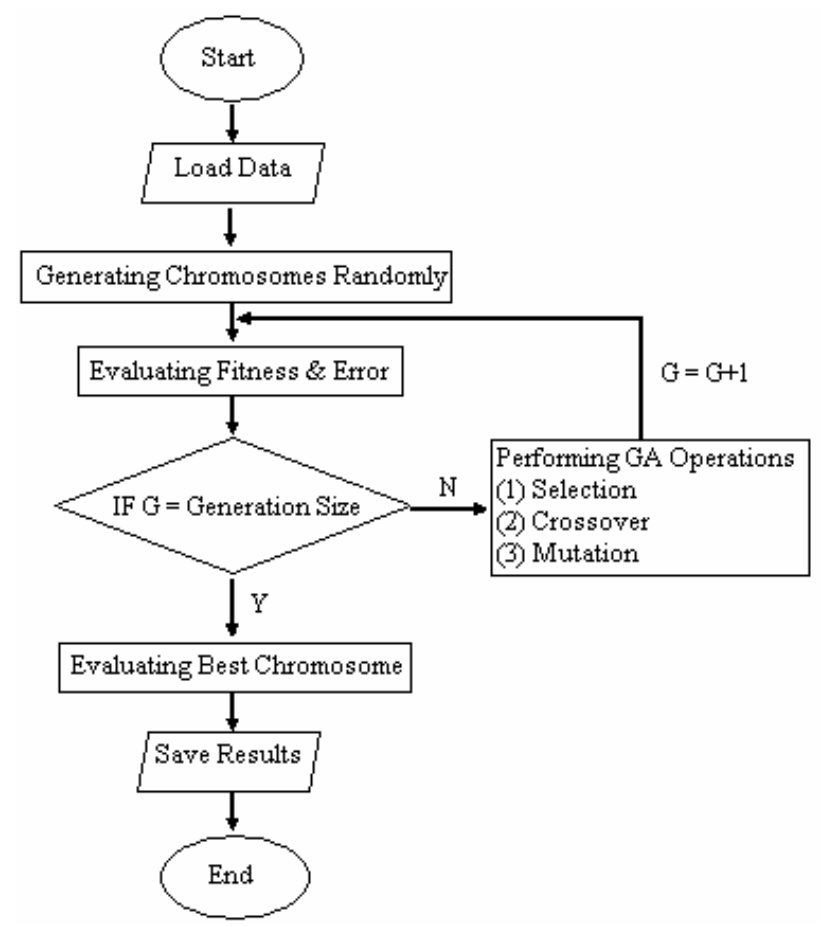

Fig.2. Basic flowchart showing the sequence of operations of the GA

\section{ADVANTAGES OF THE PROGRAM}


The advantages of the proposed program are summarized in this section:

- The developed program overcomes the spectral leakage problem of the FFT which happens when the sampled period (Tsamp) isn't an integer multiple of the fundamental (Tfund). The program was tested at Tsamp equals $(0.85,1.75,2.5)$ Tfund and high accuracy was obtained.

- The program can deal with signals containing harmonics which are not an integer multiple of the fundamental frequency and generates the Fourier series for it.

- The program has the ability to generate the Fourier series for different shape waveforms (square, triangle... etc).

- The running speed was enhanced by transforming the file written in Matlab to an executable (.exe) file.

\section{TEST CASES}

To check the applicability and accuracy of the new developed program simulated waveforms were used. Each of the simulated waveforms was sampled at different sampling frequencies. The following 2 cases are presented here to demonstrate the success and the advantages of the new program.

\section{$1^{\text {st }}$ Test Case}

This case is used to show the overcoming of the spectral leakage problem which imposes on the sampled period (Tsamp) to be an integer multiple of the fundamental period (Tfund). The simulated waveform used is for the line current of a $3 \varphi, 6$-pulse rectifier as shown in Fig. 3.

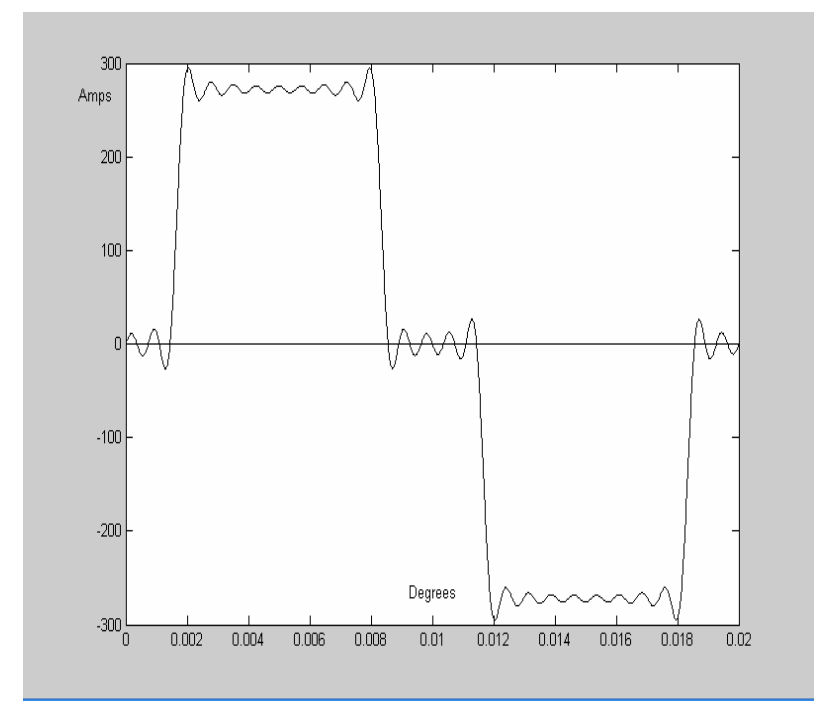

Fig.3. The Line Current of a 3- $\varphi$, 6-pulse Rectifier 
This waveform contains $\left(1,5,7,11,13,17,19,23\right.$ and $\left.25^{\text {th }}\right)$ harmonic with a fundamental freq. equals $50 \mathrm{~Hz}$. So Tfund $=1 / 50=0.02 \mathrm{sec}$.

The Tsamp used equals $0.017 \mathrm{sec}$ (i.e. Tsamp $=0.85 * 0.02=0.017 \mathrm{sec}$ ).

A comparison between the results obtained by the program and the simulated waveform values is given in Table 1. The percentage error in the harmonic magnitude is also given in the last column of the table. The 18 estimated harmonic parameters shown in Table 1 were obtained with very high accuracy, the running time of the program was $124 \mathrm{sec}$. A much faster results could be obtained with $5 \%$ max error.

\section{Table 1}

\begin{tabular}{|l|l|l|l|l|l|}
\hline \multirow{2}{*}{ Freq. } & \multicolumn{3}{|l|}{$\begin{array}{l}\text { Simulated } \\
\text { waveform }\end{array}$} & \multicolumn{3}{l|}{$\begin{array}{l}\text { Best result obtained by } \\
\text { the program }\end{array}$} & $\begin{array}{l}\text { Percentage } \\
\text { error }\end{array}$ \\
& Mag. & Phase & Mag. & Phase & \\
\hline 1 & 300 & 0 & 299.1 & 0 & 0.3 \\
\hline 5 & 60 & 180 & 61 & 178.85 & 1.6 \\
\hline 7 & 42.87 & 180 & 44 & 178.52 & 2.6 \\
\hline 11 & 27.27 & 0 & 28 & 0.4 & 2.6 \\
\hline 13 & 23.07 & 0 & 23 & 0 & 0.3 \\
\hline 17 & 17.64 & 180 & 18 & 177.28 & 2 \\
\hline 19 & 15.78 & 180 & 16 & 180 & 1.4 \\
\hline 23 & 13.05 & 0 & 12 & 0.23 & 8 \\
\hline 25 & 12 & 0 & 12 & 4.56 & 0 \\
\hline
\end{tabular}

\section{$2^{\text {nd }}$ Test Case}

This case shows the ability of the program to analyze signals having harmonics which aren't multiple of the fundamental frequency. The harmonics in the simulated waveform are: $3.5,5$, $7.7,9,11.3,13,15$, and $17^{\text {th }}$. Fig. 4 shows the waveform used in this case.

Table 2 is a comparison between the result obtained by the program and the simulated waveform. The running time of the program is $106 \mathrm{sec}$ (1.76minutes). The total no. of variables identified are 18 . 


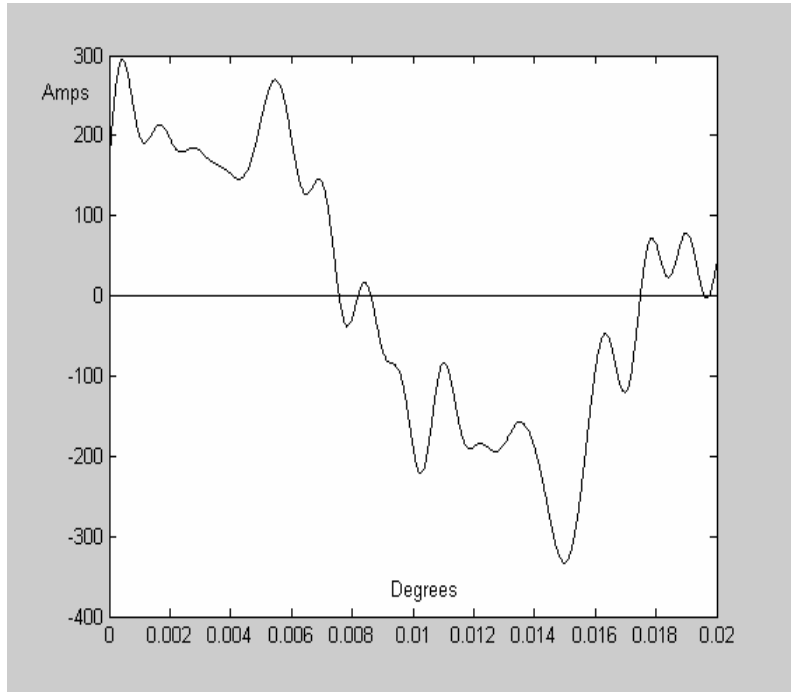

Fig.4. The waveform used in case 2

Table 2

\begin{tabular}{|c|c|c|c|c|c|}
\hline Freq. & 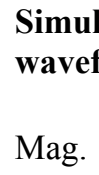 & $\begin{array}{l}\text { ed } \\
\text { Phase }\end{array}$ & $\begin{array}{l}\text { Best } r \\
\text { by the } \\
\text { Mag. }\end{array}$ & $\begin{array}{l}\text { obtained } \\
\text { gram } \\
\text { Phase }\end{array}$ & $\begin{array}{l}\text { Percentage } \\
\text { error }\end{array}$ \\
\hline 1 & 220 & 30 & 218.5 & 30.04 & 0.68 \\
\hline 3.5 & 50 & 50 & 49.5 & 50.29 & 1 \\
\hline 5 & 25 & -20 & 25 & -19.7 & 0 \\
\hline 7.7 & 35 & 0 & 35 & -0.84 & 0 \\
\hline 9 & 27 & 30 & 26.5 & 28.61 & 1.85 \\
\hline 11.3 & 21 & 60 & 21.5 & 61.36 & 2.38 \\
\hline 13 & 13 & -50 & 12.5 & -49.8 & 3.84 \\
\hline 15 & 23 & 0 & 23 & 1.37 & 0 \\
\hline 17 & 10 & 20 & 10 & 23.6 & 0 \\
\hline
\end{tabular}

The GA parameters used in both test cases are as follows:

- Population size: 100

- Generation size : 80

- Selection type: Rank selection with Elitism.

- Coding: Real numbers.

- Crossover operator: Single point crossover. 
- Crossover probability: 0.9

- Modified Mutation prop: 0.8

The running time differs according to:

- Generation size

- Sampling frequency

- Sampling period

- The no. of variables to be solved.

\section{CONCLUSION}

The developed program is a new method to identify harmonics using the GAs. Due to its high accuracy, the program can be used in different voltage or current levels (power system or communication field). As have been shown from the test cases the results obtained by the program were very accurate.

The user can interface with the program and so can change the GAs parameters. While the time consumed in program running is a drawback of this technique. An effort is being done to decrease the running time of the program so it can be used in on-line harmonic identification.

\section{REFERENCES}

[1] Sergio Herraiz, Luis Sainz and Jordi Clua, "Review of Harmonic Load Flow Formulations"; IEEE Trans. On Power Delivery, Vol.18, No. 3, July 2003.

[2] Aly A. Mahmoud and Richard D. Shultz, "A Method for analyzing harmonic distribution in A.C power systems"; IEEE Trans. On Power Apparatus and Systems, Vol. Pas-101, No. 6, June 1982.

[3] J. Arrillaga and C.D Callagnam, "Three phase AC-DC load and harmonic flows"; IEEE Trans On Power Delivery, Vol. 6, No. 1, January 1991.

[4] Manuel Valcárcel and Julio G. Mayordomo, "Harmonic power flow for unbalanced systems"; IEEE Trans. On Power Delivery, Vol. 8 No. 4, October 1993.

[5] Rennard, J.-Ph. (2000). Introduction to Genetic Algorithms. Retrieved May/2005, from http://www.rennard.org/alife/english/gavgb.pdf

[6] Maamar Bettayeb and Uvais Qidwai, "A Hybrid Least Squares-GA-Based Algorithm for Harmonic Estimation"; IEEE Trans. On Power Delivery, Vol. 18, No. 2, April 2003.

[7] Wael M. Al-Hasawi, Hosam K. M. Youssef and Khaled M. El Naggar, "A Genetic Algorithm for On-Line Identification and Tracking of Power System Harmonics"; Proceedings at the LASTED International Conference, Power and Energy systems, September 19-22, 2000 Marbella, Spain.

[8] L.L. Lai, W.L. Chan, C.T. Tse and A.T.P. So, "Real-Time Frequency and Harmonic Evaluation using Artificial Neural Networks"; IEEE Trans. On Power Delivery, Vol. 14 No. 1, January 1999.

[9] T. Ghose, S.K. Goswami and K. Basu, "Solving Capacitor Placement Problem in Distribution Systems Using Genetic Algorithm" Electric Machines \& Power Systems Journal, VOL.27, 1999, pp.429-44. 
[10] Reformate M., Kuffel E., Woodford D. and Pedrcyz W., "Application of Genetic Algorithms for Control Design in Power Systems" Generation, Transmission and Distribution IEE- Proce., Vol.145-4, July 1998, pp.345-354 